\title{
Mini-Magnetospheric Plasma Propulsion (M2P2): High Speed Propulsion Sailing the Solar Wind
}

\author{
Robert Winglee ${ }^{1}$ John Slough ${ }^{2}$, Tim Ziemba ${ }^{2}$, and Anthony Goodson ${ }^{3}$ \\ ${ }^{1}$ Geophysics Program, University of Washington, Seattle WA 98195-1650, \\ ph: 1-206-685-8160,e-mail: winglee@geophys.wasington.edu \\ ${ }^{2}$ Department of Aeronautics and Astronautics, University of Washington, Seattle WA 98195-2250 \\ ${ }^{3}$ The Boeing Corporation, P. O. Box 3999, MS 84-33, Seattle WA 98124
}

\begin{abstract}
Mini-Magnetospheric Plasma Propulsion (M2P2) seeks the creation of a magnetic wall or bubble (i.e. a magnetosphere) that will intercept the supersonic solar wind which is moving at $300-800 \mathrm{~km} / \mathrm{s}$. In so doing, a force of about $1 \mathrm{~N}$ will be exerted on the spacecraft by the spacecraft while only requiring a few $\mathrm{mN}$ of force to sustain the minimagnetosphere. Equivalently, the incident solar wind power is about $1 \mathrm{MW}$ while about $1 \mathrm{~kW}$ electrical power is required to sustain the system, with about $0.25-0.5 \mathrm{~kg}$ being expended per day. This nominal configuration utilizing only solar electric cells for power, the $\mathrm{M} 2 \mathrm{P} 2$ will produce a magnetic barrier approximately $15-20 \mathrm{~km}$ in radius, which would accelerate a $70-140 \mathrm{~kg}$ payload to speeds of about $50-80 \mathrm{~km} / \mathrm{s}$. At this speed, missions to the heliopause and beyond can be achieved in under 10 yrs. Design characteristics for a prototype are also described.
\end{abstract}

\section{INTRODUCTION}

Missions to travel to the outer rim of the solar system and beyond is no easy matter. The heliopause, which represents the boundary between the solar wind and the interstellar wind (Suess, 1990) is thought to be at about 140 $+/-20 \mathrm{AU}$, with $1 \mathrm{AU}=1.5 \times 10^{8} \mathrm{~km}$ being the distance from the Sun to the Earth (Baranov and Malama, 1993; Steinolfson et al., 1994; Linde et al., 1998). The solar wind itself moves out from the Sun with an average speed of 300 to $800 \mathrm{~km} / \mathrm{s}$ (and on occasions even to $1000 \mathrm{~km} / \mathrm{s}$ ) and experiences essentially no deceleration until the termination shock, which is at approximately $80+/-10 \mathrm{AU}$. It is at this region where the flow changes from supersonic to subsonic. This deceleration is produced by interactions from reflected solar wind particles and transmitted interstellar particles from the interstellar medium as well as from interactions from neutrals from the interstellar medium.

For optimum scientific return, a spacecraft mission to these regions is presently required to be performed within a 10 -yr period after launch. However, the distances involved are huge. For example Voyager 1, which was launched in 1977 with a present speed of about $17 \mathrm{~km} / \mathrm{s}$ (twice the speed of the space shuttle) is only at about $70 \mathrm{AU}$ at this time and is expected to encounter the termination shock in the next few years. The high speed of Voyager 1 has in part been facilitated by gravitational boosts from its planetary encounters but it is still not expected to cross the heliopause for another $20 \mathrm{yrs}$. A 10 -yr mission to these regions requires a minimum speed of about $50 \mathrm{~km} / \mathrm{s}$ or equivalently $10 \mathrm{AU} / \mathrm{yr}$.

A critical factor in determining the speed of a spacecraft is the exit velocity of the propellant. For chemical thrusters the maximum exit velocity is only about $3-5 \mathrm{~km} / \mathrm{s}$. Because the fuel-to-payload ratio goes as the exponential of change in spacecraft velocity to the propellant velocity, a spacecraft cannot economically attain a speed of $50 \mathrm{~km} / \mathrm{s}$ by this means. In order to increase speeds beyond existing chemical systems, the propellant speed must be increased substantially and the corresponding high temperatures means that the propellant must be in the form of a plasma. However, the energy requirements to produce sufficient plasma to propel a spacecraft to $50 \mathrm{~km} / \mathrm{s}$ are enormous and cannot be sustained by solar electric cells alone. Nuclear power could be used but would greatly increase the mass

CP504, Space Technology and Applications International Forum-2000, edited by M. S. El-Genk

02000 American Institute of Physics 1-56396-9 19-X/00/\$17.00

962 
and cost of the spacecraft and at the same time pose a potential environmental hazard. The only other alternative is to utilize ambient energy through some form of sail technology. Solar sails have been proposed where the spacecraft picks up the momentum from the reflection of light. However, such sails face substantial technological difficulties in attaining sufficiently light material and being able to deploy the material over large distances in space.

An alternative that is described here is to tap the energy of the solar wind and as such would provide a specific impulse of $3-8 \times 10^{4} \mathrm{~s}$. Initial proposals to harness this were through magnetic sails or magsail (Zubrin, 1993 and references therein). While different variants have been discussed, the basic concept was to deploy a superconducting magnet with a radius of $100-200 \mathrm{~km}$ to attain accelerations of the order of $0.01 \mathrm{~m} / \mathrm{s}^{2}$. The use of superconducting material would essentially eliminate all power requirements, except for initially setting up the currents and maintaining any cryogenic systems. However, the drawback of such a system was that it was fairly massive (of the order of a few metric tons) and the physical construction and cost of such a system presently limit its usage from technical and/or economical viewpoints.

The Mini-Magnetospheric Plasma Propulsion (M2P2) described here is analogous to the solar and magnetic sails in that it seeks to harness ambient energy in the solar wind to provide thrust to the spacecraft. However, it represents major advances in several key areas. First, the M2P2 will utilize electromagnetic processes (as opposed to mechanical structures) to produce the obstacle or sail. Thus, the technical and material problems that have beset existing sail proposals are removed from the problem. Second, because the deployment is electromagnetic large scale cross-sections (15 - $20 \mathrm{~km}$ for a prototype version) for solar wind interactions can be achieved with low weight $(<50 \mathrm{~kg}$ for the device) and low power $(<3 \mathrm{~kW})$ requirements. Third, the M2P2 system acts similar to a balloon in that it will expand as the solar wind dynamic pressure decreases with distance from the sun. As such it will provide a constant force surface (as opposed to a mechanical structure that provides a constant area surface) and thereby provide almost constant acceleration to the spacecraft as it moves out into the solar system.

The physical principles and system requirements are discussed in Section 2 for a spacecraft that could move out of the solar system with speeds of $50-80 \mathrm{~km} / \mathrm{s}$ after an acceleration period of about three months. Detailed numerical results from fluid simulations of the M2P2 system are given in Winglee et al. (1999). Section 3 describes developments in the making of a prototype of the M2P2 and the expected performance of such a system. A summary of results is given in Section 4.

\section{CONCEPT DESCRIPTION}

The basic objective of the M2P2 system is to deflect the solar wind particles by a large magnetic bubble or minimagnetosphere whose field lines are attached to the spacecraft. As the charged particles of the solar wind are reflected by the magnetic field the force that they exert is transmitted along the fields lines to the spacecraft to produce its acceleration. In the simplest case in Figure 1a, the magnetic field is produced solely by a solenoid coil. In this case, the intensity of the magnetic field decreases as $\mathrm{R}^{-3}$ so that within about 10 coil radii the interaction the magnetic field is essentially zero and the interaction region with the solar wind is very restricted, and hence inefficient. However, with the injection of plasma, the plasma can drag the magnetic field outwards once it moves into a region where the thermal and/or dynamic plasma pressure exceeds the magnetic pressure. As a result the magnetic field can fall off very much more slowly than for a simple dipole, thereby facilitating much more efficient coupling to the solar wind as illustrated in Figure $1 \mathrm{~b}$.

This expansion of magnetic field by injection of plasma is seen in a variety of space plasmas. Examples include the formation of the heliopause itself, where the solar magnetic field is stretch out to $140 \mathrm{AU}$ and are sufficiently strong to modulation the cosmic ray flux as a function of solar cycle. Closer to the Earth, magnetic clouds which associated with coronal mass injections are seen to erupt from the solar surface and seen to increase in size well beyond that of the Sun. Similarly the size of Jovian magnetosphere appears inflated relative to the terrestrial magnetosphere due to plasma from Io and its acceleration though the fast rotation of Jupiter.

The importance of the plasma injection can be also be seen from the following arguments. A dipole magnetic field decreases as $\mathrm{R}^{-3}$. If the plasma energy density where made sufficiently high that it was able to freely expand and 


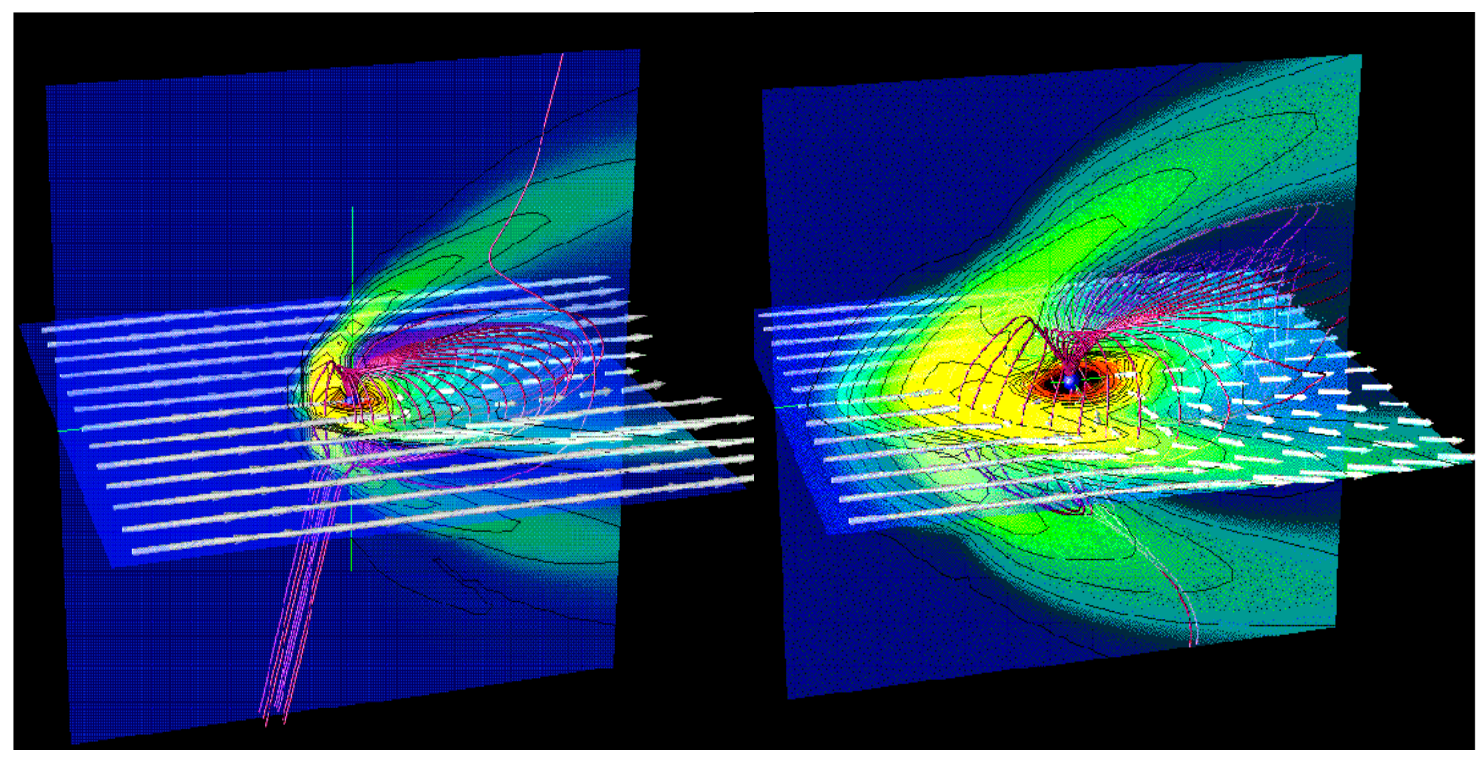

FIGURE 1. (a) The deflection of the solar wind by a simple dipole. (b) Enhanced interaction with the solar wind produced by the inflation of the magnetic field as plasma is injected into the magnetic field. The plasma flow is shown by the arrows with the size of the arrows being proportional to plasma speed. Contours of the plasma pressure are shown in the equatorial plane and in the noon-midnight meridian. The formation of a bow shock as the solar wind interacts with the magnetosphere is seen as a jump in the plasma pressure (yellow regions) around the magnetosphere. The magenta lines indicated some of the field lines attached to the magnetosphere.

carry the frozen-in magnetic field out with it then the magnetic field strength would decrease as $\mathrm{R}^{-2}$. However due to the interaction with the solar wind this plasma is contained or compressed so that current sheets are formed within the plasma and the magnetic field decreases as $\mathrm{R}^{-1}$. The numerical simulation results of Winglee et al. (1999) verify this result.

The size for an effective the mini-magnetosphere can be estimated as follows. If is assumed that the dragging of the field occurs very close to the coil (i.e., where speed of the injected plasma $\left(\mathrm{V}_{\text {plas }}\right)$ is close to the Alfven speed $\left(\mathrm{V}_{\mathrm{A}}\right)$ near the magnet, then assuming a $\mathrm{R}^{-1}$ fall off in the magnetic field then the size of the mini-magnetopause $\left(\mathrm{R}_{\mathrm{MM}}\right)$ would be given by

$$
\mathrm{R}_{\mathrm{MM}} \sim \mathrm{R}_{\text {coil }}\left(\mathrm{B}_{\text {coil }} / \mathrm{B}_{\mathrm{MP}}\right)
$$

where $\mathrm{B}_{\mathrm{MP}}$ is the field strength so that the magnetic pressure balances the solar wind pressure. For a solar wind density of $6 \mathrm{~cm}^{-3}$ and a speed of $450 \mathrm{~km} / \mathrm{s}$, the solar wind dynamic pressure is equivalent to $2 \mathrm{nPa}$ and a $\mathrm{B}_{\mathrm{MP}}=50$ $\mathrm{nT}$ is sufficient. For a mini-magnetosphere extending to $15-20 \mathrm{~km}$, the force exerted by the solar wind would be about $2.5-5 \mathrm{~N}$ (and about a MW of incident power). To produce this size magnetosphere for a coil radius of 10 $\mathrm{cm}$, the minimum field strength that is required is about $100 \mathrm{G}$.

If $\mathrm{V}_{\text {plas }}<\mathrm{V}_{\mathrm{A}}$, the coil magnetic field strength has to be increased by approximately the ratio $\mathrm{V}_{\mathrm{A}} / \mathrm{V}_{\text {plas }}$. Because the Alfven speed depends on the plasma density, the plasma that is supporting the mini-magnetosphere does not have to be energetic if it is sufficiently dense. As a consequence, the system attains a momentum and power leverage in effect by exchanging or reflecting high energy solar wind plasma with the lower energy plasma supporting the minimagnetosphere. 


\begin{abstract}
A PROTOTYPE
In order to produce coupling with the solar wind over a region of $15-20 \mathrm{~km}$, the M2P2 system has basically three components : (1) a relatively strong $(\sim 700 \mathrm{G})$ magnetic field on the spacecraft with the coil radius being about 10 $\mathrm{cm}$, (2) plasma source to inflate the magnetic field with the plasma having an energy of a few $\mathrm{eV}$ and a density of the order of $5 \times 10^{13} \mathrm{~cm}^{-3}$ for $\beta \sim 1-4 \%$ (or equivalently $\mathrm{V} / \mathrm{V}_{\text {plas }} \sim 5-10$ ) and (3) a power source sufficient to generate the magnetic field and plasma. For a $2.5 \mathrm{~cm}$ plasma source, the helicon can output several tends of $\mathrm{mN}$ of thrust for only about a $\mathrm{kW}$ of power.

There are only a few plasma sources that work in the presence of a strong magnetic field, and even fewer capable of producing the high density required. Plasmas generated using electrodes cannot tolerate the high heat load at the high energy densities. There are however inductively produced plasma sources (e.g. helicons) that not only produce high-density discharges but do it in the presence of kilogauss magnetic fields (Miljak and Chen, 1998; Gilland et al., 1998, Conway et al., 1998). It is for this reason that the prototype being developed at the University of Washington uses a helicon. The other advantage of the helicon is that it forms a closed system in the sense that the plasma is created on a field-line that is contained within the vacuum chamber and does not come into contact with the walls of the magnet or the plasma source.
\end{abstract}

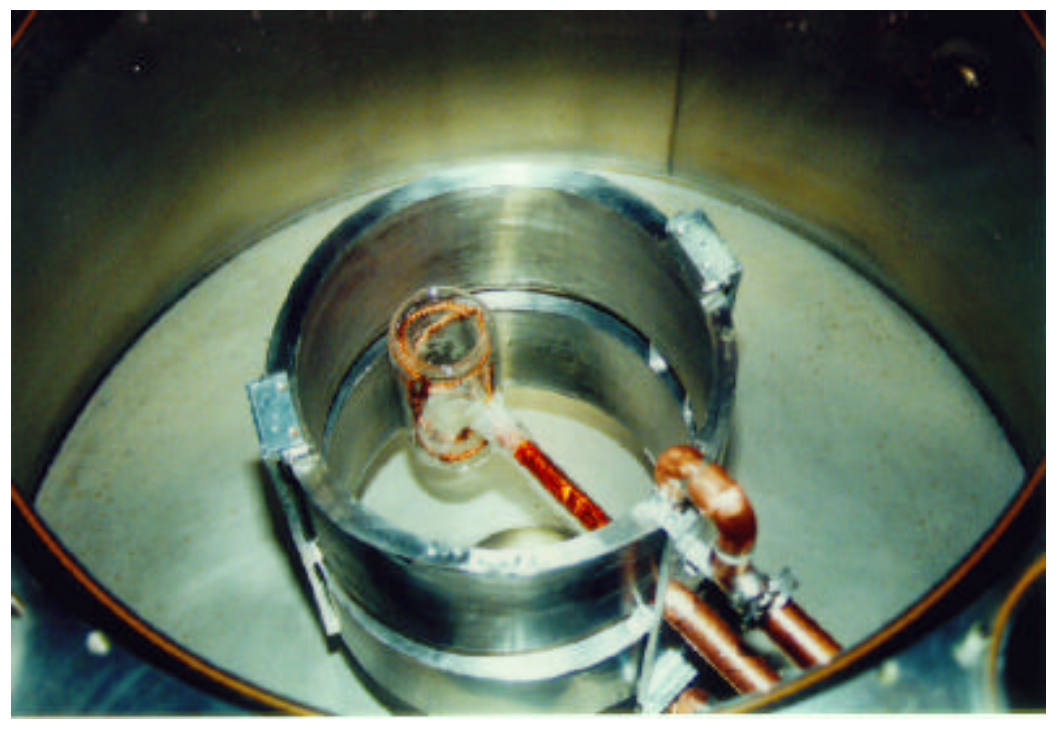

FIGURE 2. The M2P2 prototype in a small $(1 \mathrm{~m})$ vacuum chamber at the University of Washington. The magnets are encased in a stainless steel jacket so that will not be shorted by the plasma generated within the helicon (for a close-up see Figure 3). Gas and electrical feeds are passed through the copper pipes on the lower right of the figure.

A picture of the prototype is shown in Figure 2. Gas is fed into the vacuum chamber $(1 \mathrm{~m}$ diameter) through the copper pipes in the lower right part of the figure, and enters into the center of the quartz tubes $(5 \mathrm{~cm}$ diameter) seen in the center to the figure. Within the quartz tubes a Nagoya type III antenna is embedded to produce the radio frequency heating of the gas to create the plasma needed to support the mini-magnetosphere. The jacketing of the antenna on both sides by quartz tubes ensures that it is electrically isolated from the plasma. The magnetic field coils $(20 \mathrm{~cm}$ diameter) are encased in a stainless-steel jacket to avoid potential shorting by generated plasma. The plasma source is placed off-axis to ensure that the plasma is created on closed field-lines. The electrical feeds for the helicon and magnets are run through the copper pipes. 


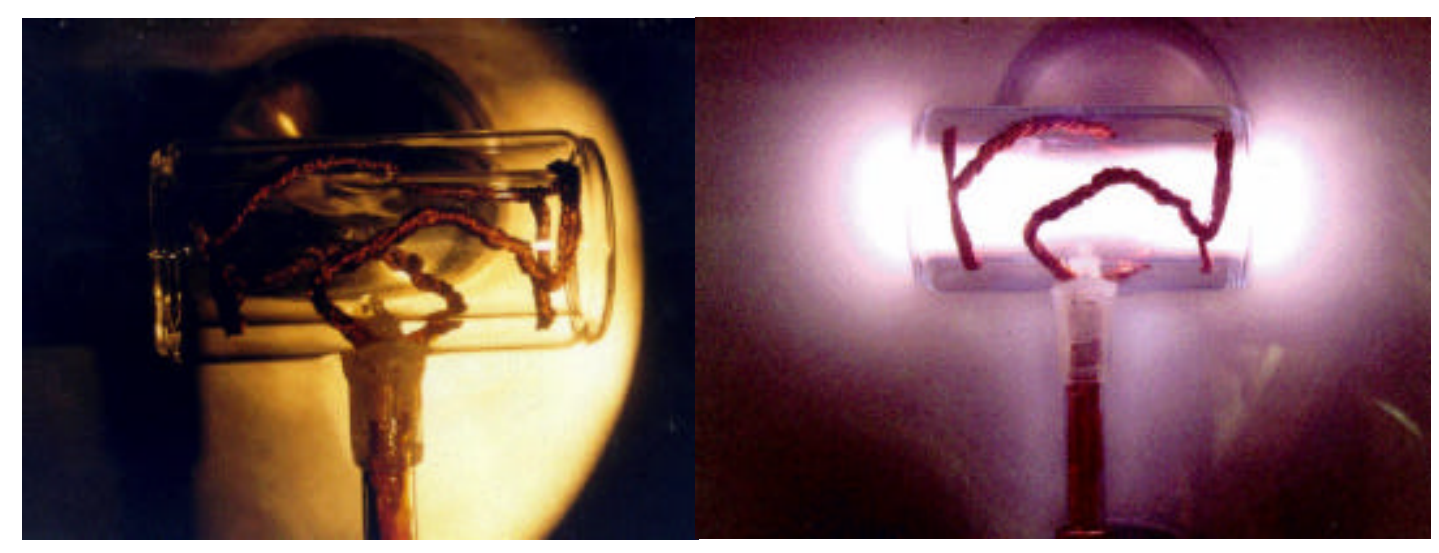

FIGURE 3. (a) Close up of the helicon in the vacuum chamber without the magnets attached. (b) Inductively coupled, highdensity helium plasma discharge in the helicon. Approximately, $1 \mathrm{~kW}$ of RF power at $19 \mathrm{MHz}$ was coupled to the plasma using a Nagoya type III antenna (copper braid in picture). The antenna is enclosed in an annular quartz envelope isolating it from the vacuum and plasma. The tube attached to the antenna enables the propellant and RF power to be delivered remotely.

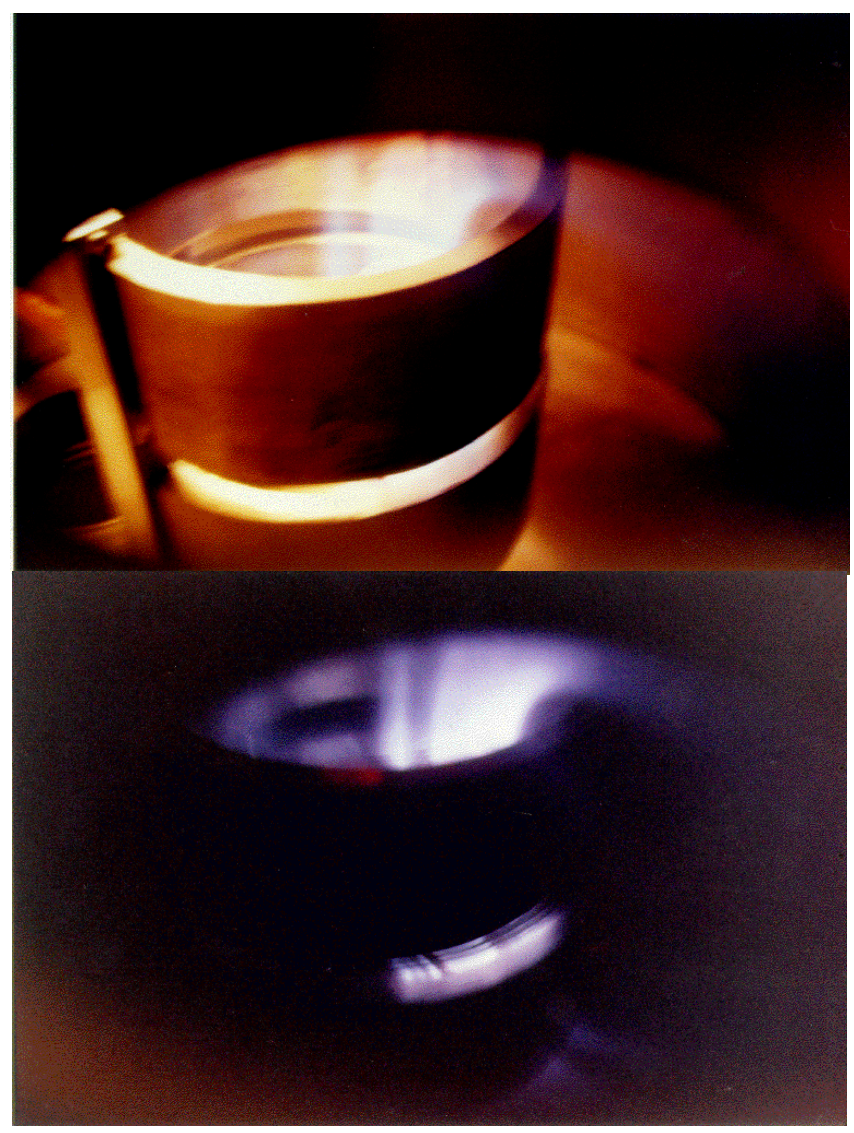

FIGURE 4. The helicon being operated with magnets operating at $300 \mathrm{G}$ with the chamber back-filled with argon at $5 \mathrm{mTorr}$. (a) The chamber illuminated by external lighting and (b) image of the plasma emissions alone. It is seen that the plasma trajectory tracks around the field lines to produce a loop-like structure faintly visible on the right hand side. 
The performance and efficiency of the helicon source in creating plasma and moving magnetic field within the chamber are being evaluated at this time. Initial images are shown in Figure 3 of the plasma production by the helicon but without the magnets attached. The light emissions show the production of a dense column of plasma, which then expands as it moves out of the quartz tube. Figure 4 shows the difference when the solenoid coils are present, and producing $300 \mathrm{G}$ fields. In this case, there is still strong plasma production but as it exits the helicon, the plasma is turned by the magnetic field to produce a loop-like structure to the right and which extends close to the chamber walls. This type of structure is one of the primary goals of the M2P2 system.

\section{SUMMARY}

In order to explore the outer solar system and nearby interstellar space spacecraft will have to travel at speeds in excess of $50 \mathrm{~km} / \mathrm{s}$. The power requirements needed to obtain such high speeds are much higher than can be presently supported by solar electric propulsion. Thus some other means of powering spacecraft must be developed. In this paper we have investigated one such system where energy from the solar wind is used to propel the spacecraft. This system which we call Mini-Magnetospheric Plasma Propulsion (M2P2) seeks to inflate a large magnetic bubble around the spacecraft to deflect and thereby pickup the momentum from the solar wind particles which are traveling at speeds of 350 to $800 \mathrm{~km} / \mathrm{s}$. A prototype for the device, consisting of a helicon plasma source inserted asymmetrically in a moderately strong magnetic field has been constructed in a laboratory vacuum chamber. Because the energy of the plasma used to produce the inflation is very much less than that of the solar wind, it is expected that only a few $\mathrm{mN}$ of force and a few $\mathrm{kW}$ of power is needed to sustain the magnetosphere, while intercepting nearly a $\mathrm{N}$ of force and a MW of power from the solar wind for a specific impulse of $3-8 \times 10^{4} \mathrm{~s}$.

\section{ACKNOWLEDGEMENTS}

This work was supported by a grant from NASA's Institute for Advance Concepts 07600-010, NSF Grant ATM9731951 and by NASA grants NAG5-6244, NAG5-8089 to the Univ. of Washington. The simulations were supported by the Cray T-90 at the San Diego Supercomputing Center which is supported by NSF.

\section{REFERENCES}

Baranov, V. B. and Malama, Y. G., "Model of the solar wind interaction with the local interstellar medium: Numerical solution of the self-consistent problem," J. Geophys. Res., 1993, 98, 15157.

Conway, G. D., Perry, A. J. and Boswell, R. W., "Evolution of ion and electron energy distributions in pulsed helicon plasma discharges," Plasma Sources, Sci. and Tech., 1998, 7, 337.

Linde, T. J., Gombosi, T. I., Roe, P. L., Powell K. G. and DeZeeuw, D. L., "Heliosphere in the magnetized local interstellar medium: results of a three-dimensional MHD simulation," J. Geophys. Res., 1998, 103, 1889.

Leipold, M., Borg, E., Lingner, S. , Pabsch, A. , Sachs, R. and Seboldt, W. , "Mercury orbiter with a solar sail spacecraft, " Acta Astronautica, 1995, 35, 635.

Miljak, D. G.,and Chen, F. F. "Density limit in helicon discharges, ” Plasma Sources, Sci. and Tech., 1998, 7, 537.

Steinolfson, R. S., Pizzo, V. J. and Holzer, T., "Gasdynamic models of the solar wind/interstellar medium interaction," Geophys. Res. Lett., 1994, 71, 245.

Suess S.T., "The heliopause," Rev. Geophys., 1990, 28, 97-115.

Weaver, H A, "Comets," Astronomical Society of the Pacific Conference Series., 1998, 143, pp. 213-26.

Weissman, P. R., "The Kuiper belt," Annual Rev. Astron. Astrophys., 1995, 33, 327.

Winglee, R. M., et al., "Modeling of upstream energetic particle events observed by WIND, " Geophys. Res. Lett., 1996, 23, 12,276,.

Winglee, R. M., Slough, J., Ziemba, T., and Goodson, A., "Mini-magnetospheric plasma propulsion: Tapping the energy of the solar wind for spacecraft propulsion, " J. Geophys. Res., submitted, 1999.

Zubrin, R. M., “The use of magnetic sails to escape from low Earth orbit,” J. British Interplanetary Society, $1993,46,3$. 